\title{
Does 3D Wound Mapping Software Compare to Expert Opinion in Determining Burn Wound Area?
}

\author{
Edmund Farrar, Ojas Pujji, Prof. Steven Jeffery
}

Department of Burns and Plastic Surgery, University Hospital Birmingham, Birmingham, United Kingdom

\section{Introduction}

New technologies in the field of burn wound and scar assessment are continually being evaluated. Accurate estimation of total body surface area (TBSA) burn wound is of paramount importance in fluid resuscitation to prevent complications which are associated with morbidity and mortality. Estimating the TBSA is performed by a multitude of different methods, however a gold standard would be ideal. The aim of this study was to compare the estimation of burned TBSA \% between 3D photography by Panasonic FZ-M1 Toughpad in conjunction with WoundCare Lite software and expert opinion volunteered by burns doctors.

\section{Methods}

Two life sized mannequins were used to simulate burns; an adult and a child. The burn was drawn to mimic real life burn patterns. Burns were measured prior to specialist assessment on the $3 D$ camera. Burns doctors were asked to estimate the TBSA $\%$ of the burn. 10 burn sizes were assessed on the adult mannequin, and 8 on the child.

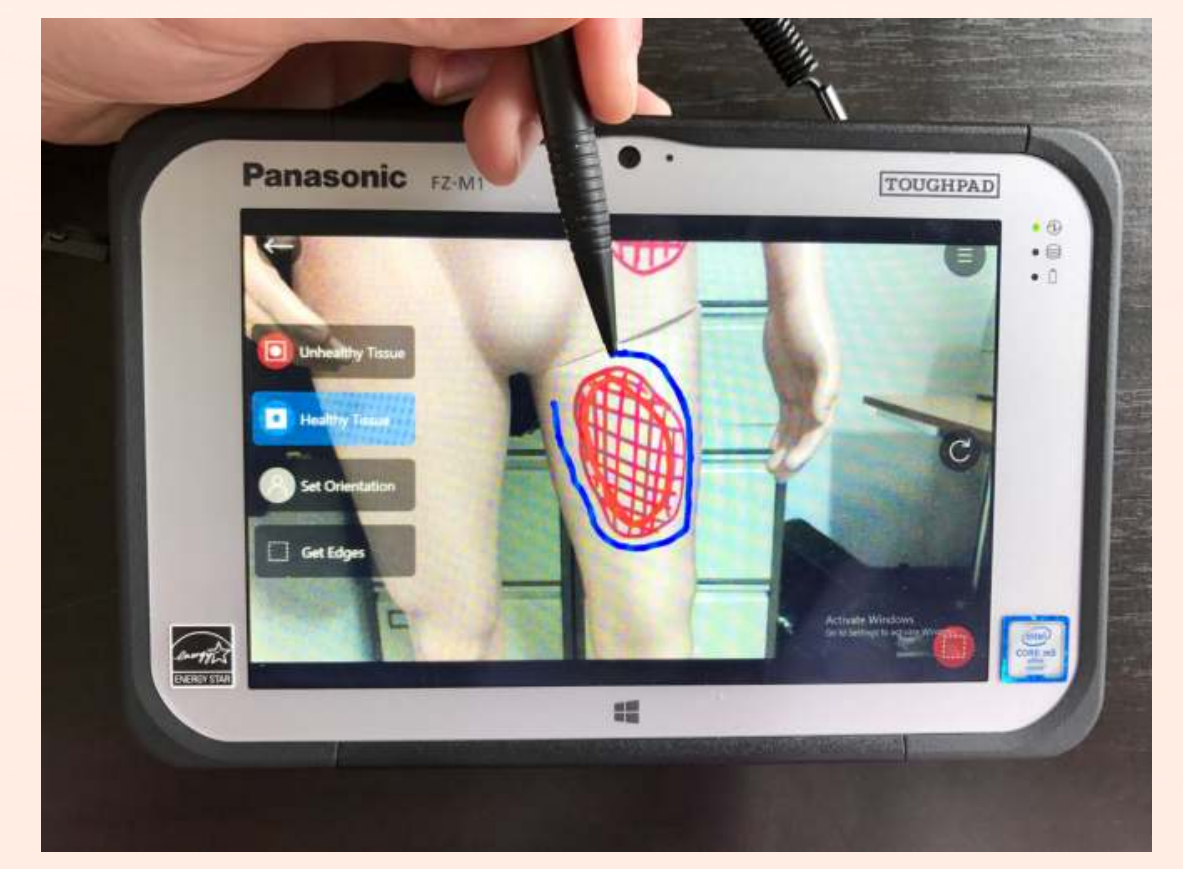

Figure 1: Image depicting how burn wound areas were measured using the WoundCare Lite software

\section{Results (1)}

Wound size as estimated from the camera varied from $0.42 \%$ to $18.23 \%$, with a mean of $5.246 \%$. Each burn was assessed by a median of 3 assessors (ranging from 2-8). Table 1 displays these results. 12 of the 18 burns $(66.67 \%)$ were assessed by at least 1 burns consultant, and of those that weren't, were assessed by a plastic surgery registrar.

\begin{tabular}{|c|c|c|}
$\begin{array}{c}\text { Grade of } \\
\text { Assessor }\end{array}$ & $\begin{array}{c}\text { No. of Assessments } \\
\text { Made (percentage) }\end{array}$ & $\begin{array}{c}\text { Median no. per } \\
\text { Burn (range) }\end{array}$ \\
\hline Consultant & $18(26.09 \%)$ & $1(0-3)$ \\
\hline Registrar & $26(37.68 \%)$ & $1.5(0-3)$ \\
\hline SHO & $25(36.23 \%)$ & $1(0-3)$ \\
\hline Total & 69 & $3(2-8)$ \\
\hline
\end{tabular}

Table 1: Demographics of assessors

Burn TBSA estimations were compared using $\operatorname{ICC}(2,1)$. This showed excellent agreement, 0.985 (95\% Cl 0.905, 0.996), between assessments of the specialist and the 3D camera (Fig. 2).

\section{Conclusions}

Figure 2: Scatterplot showing paired data measurements for specialist opinion vs the $3 \mathrm{D}$ camera

The average difference in measurements was $0.54 \%$ (LOA $0.68,1.75) \%$.
This study has demonstrated that the wound mapping software WoundCare Lite in conjunction with the Panasonic FZ-M1

Wound size estimation is of vital importance when assessing burns. Burns units have a junior doctor initially assess the burn - this stage is key in the decision of management. If a camera could be used to accurately estimate the size of burns in this early stage, there is not only potential to greatly reduce the burden on junior doctors in the initial assessment, but to reduce error and aid clinical decision making.

We acknowledge a number of drawbacks to this study. It was difficult to assess certain types of burns with the camera. These included those covering a larger area, burns over curved areas and circumferential burns. Mannequins are arguably easier to photograph and estimate burn TBSA. A human subject would move and is potentially difficult to manipulate into a suitable position for a 3D photograph. This camera only measured Burn TBSA, and not thickness. Thickness has a greater impact on the management of the burn. well with expert opinion in determining burn surface area on a mannequin. Further research is needed to establish whether this is the case in burns on patients in an acute setting.
Toughpad 3D camera compares 